\title{
NMDA receptor subunits: function and pharmacology
}

\section{Pierre Paoletti and Jacques Neyton}

Laboratoire de Neurobiologie, CNRS UMR 8544

Ecole Normale Supérieure

46 rue d'Ulm, 75005 Paris, France

E-mail: paoletti@biologie.ens.fr, neyton@biologie.ens.fr

Corresponding author: Dr. Pierre Paoletti or Dr. Jacques Neyton

\section{Summary}

NMDA receptors (NMDARs) are glutamate-gated ion channels widely expressed in the CNS that play key roles in excitatory synaptic transmission. Due to their involvement in numerous neurological disorders, NMDARs are also targets of therapeutic interest. NMDARs occur as multiple subtypes which differ in their subunit composition and in their biophysical and pharmacological properties. In particular, NMDARs contain a diversity of sites at which endogenous ligands or pharmacological agents can act to modulate receptor activity in a subunit-selective manner, and recent structural and functional data have started to reveal the molecular determinants for this subunit-selectivity. These include the binding sites for glutamate, the ion-channel pore and the recently-identified allosteric sites on the $\mathrm{N}$ terminal domain. Other potential sites yet unexplored by medicinal chemistry programs are also considered, in particular at the interface between subunits. Given the growing body of evidence that diverse brain disorders implicate different NMDAR subtypes, such as NR2B in pain or NR3A in white matter injury, there is a growing interest in exploiting the pharmacological heterogeneity of NMDARs for the development of novel NMDAR subtype-selective compounds. 


\section{Introduction}

Within the large family of excitatory ionotropic glutamate receptors (iGluRs), NMDARs constitute a sub-family identified by specific molecular composition and unique pharmacological and functional properties $[1,2]$. Of particular importance is the high permeability to calcium ions, which confers on NMDARs receptors a central role in both synaptic plasticity under physiological conditions and neuronal death under excitotoxic pathological conditions. Because they are built by heteromeric assembly from a relatively large pool of homologous subunits, NMDARs exist as diverse subtypes endowed with distinctive functional properties and patterns of expression [3]. Since the cloning of the different subunit isoforms, relating particular functions to NMDAR subtypes has been a continuous challenge [2]. In this review, we will concentrate on recent structural and pharmacological data that may help in revealing detailed NMDAR functions.

\section{Molecular organization and operation of NMDARs}

NMDARs are heteromeric complexes incorporating different subunits chosen within a repertoire of three subtypes: NR1, NR2 and NR3. There are eight different NR1 subunits generated by alternative splicing from a single gene, four different NR2 subunits $(A, B, C$ and $D)$ and two NR3 subunits $(A$ and $B)$; the NR2 and NR3 subunits are encoded by six separate genes [1]. Expression of functional recombinant NMDARs in mammalian cells requires the co-expression of at least one NR1 and one NR2 subtype. The stoichiometry of NMDARs has not yet been established definitely but the consensus is that NMDARs are tetramers that most often incorporate two NR1 and two NR2 subunits of the same or different subtypes [1]. In cells expressing NR3, it is thought that this subunit co-assembles with NR1 and NR2 to form ternary NR1/NR2/NR3 tetrameric complexes [4].

\section{Functional domains in NMDAR subunits}

NMDAR subunits all share a common membrane topology (Figure 1) characterized by (I) a large extracellular $\mathrm{N}$-terminus, (ii) a membrane region made of three transmembrane segments (TM1, 3 and 4) plus a re-entrant pore loop (M2), (iii) an 
extracellular loop between TM3 and TM4 and (iv) a cytoplasmic C-terminus, which varies in size depending on the subunit and provides multiple sites of interaction with numerous intracellular proteins $\left[1,5^{*}\right]$.

The extracellular region of NMDAR subunits (like that of other eukaryotic iGluR subunits) is organized as a tandem of 2 domains that share structural and functional homologies with two families of bacterial periplasmic proteins (Figure 1). The $\mathrm{N}$ terminal domain (NTD; first 350 amino acids) shows sequence homologies with the bacterial protein LIVBP (leucine/isoleucine/valine-binding protein) [6,7]. This domain plays an important role in subunit assembly [8]. In NR2A and NR2B, the NTD also contains binding sites for allosteric inhibitors such as $\mathrm{Zn}^{2+}$ and ifenprodil (see below). The second domain comprises the pre-TM1 region and the TM3-TM4 loop ( 150 aa each). It shows sequence homologies with the bacterial protein QBP (glutaminebinding protein) and contains the agonist-binding site. Activation of NMDARs requires the simultaneous binding of two co-agonists, glutamate and glycine (or D-serine). The agonist-binding domain (ABD) binds glycine in NR1 and NR3, whereas NR2ABDs bind glutamate $\left[9^{*}, 10^{*}\right]$.

The presence of a re-entrant loop in the transmembrane region of iGluR subunits, together with a likely tetrameric quaternary structure, has favoured the hypothesis that iGluR ionic pores are homologous to an inverted potassium channel [11]. The sequences of the regions lining the pore are highly conserved in NR2 subunits and, accordingly, permeation properties (single-channel conductance, ionic selectivity), as well as affinity for the pore blocker $\mathrm{Mg}^{2+}$, vary little among the different NR1/NR2 receptor subtypes. In contrast, incorporating the NR3 subunit markedly decreases single-channel conductance, $\mathrm{Ca}^{2+}$ permeability and $\mathrm{Mg}^{2+}$ block [4]. The NR3 pore loop significantly diverges from that of other subunits, most prominently around the $Q / R / N$ site that forms the selectivity filter of iGluRs. The sequence at this locus is NS in NR1, NN in NR2 and GR in NR3. The presence of a positively charged amino acid in NR3 is likely to be responsible for the specific permeation properties of NR3-containing receptors [12].

\section{Structural aspects of NMDAR activation}


The crystallographic studies of Gouaux and colleagues on the ABD of GluR2 (an AMPAR subunit) have markedly improved our understanding of the initial steps in iGluR activation [13]. The available structures, which now include NR1 and NR2A ABDs, have fully confirmed the predicted structural and functional homologies between the bacterial protein QBP and iGluR-ABDs [9*,14]. These latter domains show the typical two-lobe fold with ligand binding in the central interlobe cleft. Agonists stabilize a closed conformation of the cleft, while competitive antagonists prevent its closure $\left[5^{\star}\right]$. Within this overall conserved mechanism, the crystallographic structures allow identification of interesting subunit specificities. Whereas coordination of the $\alpha$-carboxylate moiety of the agonist is very much conserved in all iGluR-ABDs, coordination of the $\alpha$-amino group differs between NR2A and all other crystallized iGluR-ABDs [9*]. In NR1 and GluR2, the $\alpha$-amino group makes a salt bridge with a conserved carboxylate from lobe 2 (E705 in GluR2, D732 in NR1). In NR2A, the lateral chain of the homologous carboxylate (D731) has flipped away from the $\alpha$-amino group and the salt bridge is replaced by an $\mathrm{H}$-bond network that includes residues from both lobes. This structural rearrangement has an important pharmacological consequence: it provides space in the NR2A binding pocket for the NMDA molecule to fit. Another substitution one residue upstream in NR1 explains why glutamate does not bind the NR1-ABD. In that subunit, a bulkier tryptophan replaces a tyrosine (NR2) or leucine (GluR2) and prevents glutamate binding by steric hindrance [14]. Comparison of ligand-bound GluR2 and NR1 ABDs shows yet another difference: there are more direct inter-lobe interactions in the ligand-bound NR1-ABD than in GluR2-ABD [15]. This may explain why the NMDAR affinity for glycine $\left(E_{50} 0.1-2 \mu \mathrm{M}\right)$ is significantly higher than that of AMPARs for glutamate $\left(E_{50} 10-100 \mu \mathrm{M}\right)$. It would be interesting to explore whether a high number of interlobe interactions also controls the high affinity of NR2-ABDs for glutamate $\left(E_{50} 0.5\right.$ $5 \mu \mathrm{M})$.

In iGluRs, the mechanism that links agonist binding to channel gating is partly known and relies upon subunit dimerization. The ABDs form dimers through back-toback apposition of lobes 1 burying a large surface area (Figure 1) $\left[5^{*}, 9^{*}\right]$. Because, within a dimer, lobes 1 are "glued" together by this domain interface, closure of the ABDs upon agonist binding increases the distance between lobes 2. This, in turn, exerts some tension on the linkers connecting the $A B D s$ to the pore domain, leading 
to channel opening (activation). The tension can be relaxed by breaking the ABD dimer interface. This is the core of the mechanism of desensitization in AMPARs $\left[16^{*}\right]$.

In the heteromeric NMDAR complex, the quaternary arrangement of the subunits around the central symmetry axis will govern the dimerization possibilities. Alternating NR1 and NR2 subunits would prevent the formation of homo-dimers, whereas an NR1/NR1/NR2/NR2 arrangement would allow homo- as well as heterodimer formation. Experiments using concatenated subunits favour the homo-dimer hypothesis and thus the second type of arrangement [17]. Crystallized NR1-ABD homo-dimers have been observed under particular conditions [15]. However, upon co-crystallization of NR1 and NR2A ABDs, only hetero-dimers were obtained and functional data using engineered intersubunit disulfide bridges indicate that such NR1-NR2A heterodimers are present in the intact receptor $\left[9^{*}\right]$. These results have not yet been generalized to other NR2 subunits. The possibility remains that the functional dimerization and possibly the quaternary arrangement may depend on the type of NR2 (or NR3) present in the receptor.

\section{Pharmacology of NMDAR subtypes}

Ever since the pioneering work of Watkins and colleagues showing that $\mathrm{N}$-methyl-Daspartate selectively activates a subclass of glutamate receptors (hence coined NMDARs), extensive efforts have been made to discover potent and selective NMDAR antagonists. The 80's saw the development of the first broad-spectrum competitive antagonists and high-affinity channel blockers. The cloning of NMDAR subunits in the early 90's and the subsequent finding that NMDARs occur in vivo as multiple subtypes with distinct subunit composition renewed this initial effort and triggered an intense campaign in the pharmaceutical industry to identify receptor subtype-selective compounds. This led to the identification of a new class of compounds, exemplified by ifenprodil, which selectively inhibit receptors containing the NR2B subunit [18]. More recently, zinc, an ion naturally occurring in the brain, has also been shown to be a selective antagonist of NR2A-containing receptors when applied at low (nM) concentrations [19,20]. However, almost thirty years after the discovery of the first NMDAR antagonists, the pharmacological tools available for 
discriminating between receptor subtypes remain surprisingly limited, since organic compounds highly selective for NR2A-, NR2C-, NR2D or NR3-containing receptors are still lacking.

\section{Compounds acting at the agonist-binding domains}

The first NMDAR antagonists were competitive antagonists acting at the glutamate binding site on the NR2 subunits. They are usually conformationally-constrained amino acid derivatives containing an $\omega$-phosphonic group [21]. One of the first compound discovered, (R)-2-amino-5-phosphonopentanoate (D-AP5), remains widely used because it displays strong preference for NMDARs over all other iGluRs. These compounds show some selectivity between the different NR2 subunits (affinity ranking typically $\mathrm{NR} 2 \mathrm{~A}>\mathrm{NR} 2 \mathrm{~B}>\mathrm{NR} 2 \mathrm{C}>\mathrm{NR} 2 \mathrm{D}$ ), but the variations of affinity are modest (<10-fold; Table 1) and does not allow selective inhibition of a particular receptor subtype. The lack of subunit-selectivity likely originates from the high degree of conservation in the NR2 glutamate binding pockets. All ten residues that directly contact the glutamate molecule in NR2A are strictly conserved in the other NR2 subunits [9*]. However, a detailed comparison of the various NR2 ABDs using 3D models reveals a few subunit-specific amino acids exposed to the ligand binding cavity [22]. They are located on the edge of the glutamate binding pocket, suggesting that small antagonists, which only probe the immediate vicinity of the glutamatebinding site are not likely to display significant NR2-subtype selectivity, while larger molecules may acquire subunit selectivity through steric effects. Accordingly, the two antagonists (R)-CPP and (R)-AP7 display greater subunit selectivity (NR2A >>NR2D) than their shorter homologues PMPA and (R)-AP5 (Table 1) [23]. The Novartis compound NVP-AAM077 is another competitive antagonist with enhanced selectivity for NR2A- over NR2B-containing receptors. However, its selectivity, originally reported to be $>100$-fold [24], had been over-estimated and is in fact an order of magnitude lower ( 10-fold) [25]. In addition, it is also a powerful antagonist of NR2Cand NR2D-containing receptors [26].

In conclusion, recent years have seen promising progress towards the discovery of NMDAR subtype-selective competitive antagonists. However, none of the available compounds are truly selective yet. The recent discovery of competitive 
antagonists highly selective for GluR5 kainate receptors, despite strong sequence conservation with other iGluR members [27] should nevertheless encourage further efforts to develop novel, more selective NMDAR competitive antagonists. Finally, numerous glycine binding site competitive antagonists have been described but they show little receptor subtype selectivity (Table 1), as expected for a binding site located on NR1, a subunit present in all receptor subtypes.

\section{Compounds acting in the pore}

A large number of organic compounds inhibit NMDARs by occluding the ion channel pore [21]. These compounds are uncompetitive antagonists, because their action requires prior activation of the receptor (i.e. pore opening). Moreover, while structurally diverse, they are all positively charged and thus act in a voltagedependent manner. NMDAR pore blockers usually discriminate poorly between NMDAR subtypes. This is the case of the dissociative anaesthetics phencyclidine (PCP), TCP and ketamine, and of the clinically-used drugs memantine and amantadine (Table 2). The highly-selective NMDAR channel blocker dizolcipine (MK801 ) is more potent at inhibiting NR1/NR2A and NR1/NR2B receptors than $\mathrm{NR} 1 / \mathrm{NR} 2 \mathrm{C}$ and NR1/NR2D receptors but the difference in affinity is relatively small ( 10-fold; Table 2). A similar pattern of selectivity is also seen with bulky polyamine derivatives like the spider toxin argiotoxin-636 or $\mathrm{N}^{1}$-dansyl-spermine. Interestingly, however, these channel blockers display over 50 -fold "preference" for NR1/NR2A or NR1/NR2B compared to NR1/NR2C or NR1/NR2D receptors $[28,29]$. The structural determinants underlying this selectivity are not fully understood, but hydrophobic interactions between aromatic cycles of the blockers and hydrophobic residues lining the outer vestibule of the pore are likely to play an important role $[28,30]$. Channel blockers with a large head group might thus be useful antagonists, at least to differentiate NR2A- and NR2B- vs NR2C- and NR2D-containing receptors.

\section{Compounds acting at the NR2 N-terminal domains}

The only known organic compounds which display a high NMDAR subtype selectivity are ifenprodil and derivatives, which are selective antagonists of NR2B-containing receptors [18]. Due to the important therapeutic promise of these antagonists (see 
below), significant efforts to identify novel derivatives have been made during the last decade [31] and some compounds with affinities and selectivities greater than that of ifenprodil have been found (Table 3). However, it is only recently that the binding site for this class of molecules has been identified. As suggested by their voltageindependent and non-competitive mechanism of action, these compounds bind neither the ABDs nor the pore but the large NTD of NR2B $[32,33]$. Since all NMDAR subunits possess a N-terminal LIVBP-like domain, it is tempting to hypothesize that other molecules may bind to other NMDAR NTDs and constitute new subunit-specific allosteric inhibitors. Such a compound has already been identified: the $\mathrm{Zn}^{2+}$ ion, which binds to the NR2A-NTD with a nanomolar affinity [7,34]. $\mathrm{Zn}^{2+}$ also binds to the NR2B-NTD but with a $>100$-fold lower affinity and does not bind NR2C- or NR2DNTD $[20,35]$. Because it is concentrated and released at many glutamatergic synapses [36], $\mathrm{Zn}^{2+}$ is likely to be an endogenous allosteric modulator of NMDARs.

There are some limitations in the use of NTD-targeted allosteric inhibitors to eliminate selectively a specific NMDAR subtype (Table 3). In particular, they all act as partial antagonists (the inhibition is not total at saturating inhibitor concentrations). This phenomenon is particularly pronounced for $\mathrm{Zn}^{2+}$ acting on NR1/NR2A receptors ( $70 \%$ maximal inhibition) but also present for ifenprodil-like compounds on NR1/NR2B receptors ( 90\% maximal inhibition) [19,37]. This pharmacological "defect" might turn into a therapeutic advantage if a minimal level of NMDAR activation is required for proper brain function.

\section{Other potential sites for ligand binding}

There are other hypothetical sites where extracellular ligands could act to modulate NMDAR activity. Besides the NR1-NTD (Figure 1, site 2), the ABD dimer interface might provide another site for new allosteric modulators (Figure 1, site 3). In AMPARs, this interface binds positive allosteric modulators such as cyclothiazide and aniracetam $[16,38]$. These agents reduce AMPAR desensitization and slow channel deactivation by stabilizing the ABD interface and the closed-cleft active conformation of each $A B D$, respectively. Given the strong conservation in the architecture of the ABD dimer between NMDARs and AMPARs [9*], it is tempting to speculate that the NMDAR ABD dimer interface could also be a locus for allosteric modulation. Since NMDARs show only little desensitization, screening protocol looking at possible 
changes in deactivation kinetics may be required to identify NMDAR ABD dimer interface modulators. Another candidate site is the linker region connecting the ABDs to the transmembrane segments (Figure 1, site 4). In AMPARs, this region forms a binding site for AMPAR-selective non-competitive antagonists of the GYKI family [39]. It remains to be explored whether the homologous region in NMDARs could also bind antagonists.

\section{Triheteromeric NMDARs complicate native NMDAR pharmacology}

There is compelling evidence that NMDARs are not always simple binary assemblies of NR1 with only one type of NR2 subunit, but that some receptors can incorporate two types of NR2 subunits [1,2]. Such triheteromeric assemblies have been observed in many brain regions, in particular NR1/NR2A/NR2B in the forebrain and NR1/NR2A/NR2C in the cerebellum (see [40*]). In vivo, the NR3 subunit is also believed to form ternary complexes by co-assembling with NR1 and NR2 subunits [4]. The heterogeneity of recombinant NMDAR populations obtained when expressing three types of subunits has hampered the study of the functional properties of triheteromeric NMDARs. Recently, however, Hatton and Paoletti (2005) have been able to overcome this difficulty and isolate recombinant triheteromeric receptors using an approach combining mutagenesis and pharmacology. They showed that receptors formed by co-assembly of NR1, NR2A and NR2B retain sensitivity for submicromolar concentrations of zinc or ifenprodil, but the inhibition saturates at $\sim 20 \%$. Similarly, NR1/NR2A/NR2C receptors containing a single zincbinding NTD are inhibited by zinc with high potency but low efficacy [40*]. The fact that triheteromeric receptors show an "intermediate" sensitivity to subunit-selective modulators means that it is not possible, with the currently available pharmacological tools, to fully eliminate NR2A- or NR2B-containing receptors using zinc or ifenprodil. This is a serious limitation in attempts to relate NMDAR function to individual subunits and it is unclear that it will be possible to discover antagonists that differentiate between di- and tri-heteromeric receptors.

\section{Renaissance of NMDARs as targets of therapeutic interest}


NMDARs have always triggered an intense interest about their therapeutic potential as drug targets because there are involved in many brain disorders [41]. Traditionally, NMDARs are best known for their role in excitotoxicity, a process during which excessive glutamate release causes over-activation of NMDARs, accumulation of intracellular calcium and eventually neuronal death. Excitotoxicity occurs during cerebral ischemia (following stroke or brain trauma) and in neurodegenerative disorders such as Parkinson's and Huntington's diseases. An over-activity of excitatory pathways is also observed in epilepsy and neuropathic pain. Most NMDAR antagonists developed in the 80's and 90's to treat these disorders failed in clinical trials because of unacceptable side-effects (hallucinations, memory and motor deficits). Considered too risky, the development of NMDAR-based therapies was abandoned. Recently, however, there has been growing evidence that subunitselective NMDAR antagonists have a much improved side effect profile compared to broad-spectrum antagonists. Moreover, novel functions of NMDARs have been uncovered triggering a renewed interest in drugs targeting NMDARs.

\section{NR2B-selective antagonists}

A likely explanation for the failures of the first-generation NMDAR antagonists is their lack of subunit specificity. By targeting the agonist-binding domain (competitive antagonists) or the channel pore (channel blockers), these compounds do not discriminate between the various NMDAR subtypes. NMDAR antagonists with improved tolerability have now been identified. The most promising compounds are ifenprodil derivatives, which selectively inhibit NR2B-containing NMDARs through binding to the NR2B N-terminal domain (see above) $[18,32,33]$. A number of highly potent NR2B-selective antagonists show good efficacy as neuroprotectants and/or pain killers in a variety of animal models [42]. These NR2B-selective antagonists are also effective in rodent and primate models of Parkinson's disease either alone or in combination with L-DOPA treatment [41]. Encouragingly, in humans, NR2B-selective antagonists do not induce the side-effects usually seen with non-selective NMDAR antagonists, even at maximally neuroprotective doses $[41,42]$. The reasons for their better tolerability are two-fold: first, they spare NMDARs that do not contain NR2B (such as most NMDARs of the cerebellum, a region important for motor coordination); second, they are maximally effective at persistently-activated NMDARs 
and at acidic $\mathrm{pH}$, conditions encountered during excitotoxicity $[37,43]$. While the first NR2B-selective antagonists displayed off-target activity (at adrenergic $\alpha 1$ receptors in particular), second generation compounds with substantially improved safety profiles have been reported. However, NR2B-selective antagonists have not turned into approved drugs yet, because of hERG-mediated cardiotoxicity and poor oral bioavailability [21]. The situation is changing rapidly as recent screenings of large chemical libraries have yielded novel NR2B-selective antagonists, structurally unrelated to ifenprodil, which may overcome these limitations $[44,45]$.

\section{NR3A subunit and the myelin sheath}

It was generally assumed that NMDAR expression in the CNS was restricted to neurons with no (or very little) expression in glial cells. Several recent studies indicate that NMDARs are in fact present on both astrocytes and oligodendrocytes. Oligodendrocytes, the cells in the white matter that produce the myelin sheath surrounding axons, are damaged by excess of glutamate and loss of the myelin sheath is observed in multiple neurological disorders including cerebral palsy, spinal cord injury, stroke and multiple sclerosis. This excitotoxicity was thought to proceed through activation of AMPA/kainate receptors, since oligodendrocyte somata are not protected against glutamate-mediated injury by NMDAR antagonists. Three new studies now reveal that oligodendrocytes do express functional NMDARs, but only on their processes $\left[46^{*}, 47^{*}, 48^{*}\right]$. Myelin NMDARs are only weakly blocked by extracellular $\mathrm{Mg}^{2+}$ and mediate calcium accumulation in myelin during ischemia. NMDAR antagonists prevent this accumulation and protect against ischemia-induced myelin damage. Interestingly, all three studies suggest that myelin NMDARs have an unusual subunit composition with high levels of NR2C and NR3A subunits, two subunits which are known to decrease $\mathrm{Mg}^{2+}$ block. NMDARs containing NR3A (or $\mathrm{NR2C}$ ) subunits are thus potentially major therapeutic targets for preventing white matter damage. There is currently no pharmacology of NR3A but this subunit has a ligand binding profile substantially different from that of NR1 (despite the fact that both bind glycine) suggesting that it should be possible to develop NR3A-selective antagonists [10*]. However, one should keep in mind that NR3A (expressed in isolation) has an exceptionally high affinity for glycine (650-fold higher than NR1), such that, in vivo, NR3A subunits are likely to be tonically occupied by ambient 
glycine $\left[10^{*}\right]$. Therefore, only highly potent NR3A-selective competitive antagonists, capable of displacing endogenous glycine, would be therapeutically valuable. The alternative would be to develop NR3A-selective allosteric inhibitors. Intriguingly, cortical astrocytes also express NMDAR-mediated currents with low $\mathrm{Mg}^{2+}$ sensitivity $\left[49^{*}\right]$ suggesting that incorporation of NR3A subunit may be a distinctive feature of glial NMDARs.

\section{NMDAR enhancers against NMDAR hypo-function in schizophrenia}

Several lines of evidence indicate that hypo-function of NMDARs may be a key feature in major human cognitive disorders, most particularly schizophrenia. Nonselective NMDAR channel blockers (such as PCP or ketamine) disrupt memory formation and cause a schizophrenia-like syndrome in humans, recapitulating both positive and negative symptoms and cognitive impairments [50]. Transgenic mice with reduced NMDAR expression or impaired NMDAR function display behaviours related to schizophrenia [51,52]. Finally, recent genetic linkage and post-mortem studies point directly to the involvement of NMDAR dysfunctions in the pathogenesis of human psychoses $\left[53^{*}, 54\right]$. All this evidence indicates that increasing NMDAR activity should be beneficial for treating cognitive disorders. A direct activation of the receptors by glutamate site agonists, if conceivable in theory, raises immediate concerns regarding excitotoxicity. Increasing the activity at the co-agonist glycine site is a possible alternative and has shown some clinical benefit [55]. Another promising strategy may reside in developing molecules capable of enhancing NMDAR activity through binding to modulatory sites (NMDAR enhancers or positive allosteric modulators). There are two potential mechanisms through which NMDAR activity could be enhanced: first, by preventing NR2-NTD closure with compounds that would displace the endogenous ligand $\mathrm{Zn}^{2+}$ and maintain the NTD-cleft in a more open conformation (Figure 1, site 1); second, by stabilizing the channel open state (slowing of deactivation) or blocking the entry into a desensitized state with compounds binding the ABD dimer interface (Figure 1, site 3). There are thus varied opportunities to enhance NMDAR activity and the coming years should tell us if NMDARs are a viable target for the development of novel antipsychotic agents. 


\section{Conclusions}

The NMDAR complex contains several potential binding sites for extracellular modulators. In this review we have advertised the choice of the $\mathrm{N}$-terminal domains of NMDAR subunits as an interesting therapeutical target. These domains indeed bind allosteric modulators in two NMDAR subunits (NR2A and NR2B) with strong subunit selectivity and it is tempting to speculate that other selective modulators may be found that bind NTDs of other NMDAR subunits (and more generally those of other iGluRs). More knowledge on the 3D structure may help in the discovery of new active molecules. No iGluR-NTD has been crystallized so far and even the source of the selectivity of NR2A and NR2B NTDs for $\mathrm{Zn}^{2+}$ and ifenprodil remains unclear. Eagerly awaited $3 D$ structures will not only help in understanding the selectivity and the mechanism of action of the available allosteric inhibitors, but may also enable the design of new inhibitors for orphan NTDs. 


\section{References}

1. Dingledine R, Borges $\mathrm{K}$, Bowie $\mathrm{D}$, Traynelis SF: The glutamate receptor ion channels. Pharmacol Rev 1999, 51:7-61.

2. Cull-Candy SG, Leszkiewicz DN: Role of distinct NMDA receptor subtypes at central synapses. Sci STKE 2004, 2004:re16.

3. Yamakura T, Shimoji K: Subunit- and site-specific pharmacology of the NMDA receptor channel. Prog Neurobiol 1999, 59:279-298.

4. Sasaki YF, Rothe T, Premkumar LS, Das S, Cui J, Talantova MV, Wong HK, Gong X, Chan SF, Zhang D, et al.: Characterization and comparison of the NR3A subunit of the NMDA receptor in recombinant systems and primary cortical neurons. $J$ Neurophysiol 2002, 87:2052-2063.

5. Mayer ML: Glutamate receptors at atomic resolution. Nature 2006, 440:456-462.

* In this up-to-date review, the author dissects the modular organization of ionotropic glutamate receptors and, based on recent crystallographic and functional data, gives an integrated view of their molecular operation mechanism

6. Masuko T, Kashiwagi K, Kuno T, Nguyen ND, Pahk AJ, Fukuchi J, Igarashi K, Williams K: A regulatory domain (R1-R2) in the amino terminus of the $\mathrm{N}$-methyl-D-aspartate receptor: effects of spermine, protons, and ifenprodil, and structural similarity to bacterial leucine/isoleucine/valine binding protein. Mol Pharmacol 1999, 55:957-969.

7. Paoletti P, Perin-Dureau F, Fayyazuddin A, Le Goff A, Callebaut I, Neyton J: Molecular organization of a zinc binding $\mathrm{N}$-terminal modulatory domain in a NMDA receptor subunit. Neuron 2000, 28:911-925.

8. Meddows E, Le Bourdelles B, Grimwood S, Wafford K, Sandhu S, Whiting P, Mcllhinney RA: Identification of molecular determinants that are important in the assembly of N-methyl-D-aspartate receptors. J Biol Chem 2001, 276:18795-18803.

9. Furukawa $\mathrm{H}$, Singh SK, Mancusso $\mathrm{R}$, Gouaux $\mathrm{E}$ : Subunit arrangement and function in NMDA receptors. Nature 2005, 438:185-192.

* This paper presents structural and functional evidence for heteromeric dimerization between NR1 and NR2A NMDAR subunits. As a bonus, the paper shows and discusses the structure of the glutamate-bound NR2A agonist-binding site.

10. Yao Y, Mayer ML: Characterization of a soluble ligand binding domain of the NMDA receptor regulatory subunit NR3A. J Neurosci 2006, 26:4559-4566.

* This paper presents biochemical evidence showing that the agonist-binding domain of the NR3A and NR1 subunits have a distinct pharmacological profile, despite the fact that both bind glycine.

11. Wollmuth LP, Sobolevsky Al: Structure and gating of the glutamate receptor ion channel. Trends Neurosci 2004, 27:321-328.

12. Matsuda K, Kamiya Y, Matsuda S, Yuzaki M: Cloning and characterization of a novel NMDA receptor subunit NR3B: a dominant subunit that reduces calcium permeability. Brain Res Mol Brain Res 2002, 100:43-52. 
13. Armstrong N, Sun $Y$, Chen GQ, Gouaux E: Structure of a glutamate-receptor ligandbinding core in complex with kainate. Nature 1998, 395:913-917.

14. Furukawa $H$, Gouaux $E$ : Mechanisms of activation, inhibition and specificity: crystal structures of the NMDA receptor NR1 ligand-binding core. Embo J 2003, 22:2873-2885.

15. Inanobe A, Furukawa H, Gouaux E: Mechanism of partial agonist action at the NR1 subunit of NMDA receptors. Neuron 2005, 47:71-84.

16. Sun $Y$, Olson R, Horning M, Armstrong N, Mayer M, Gouaux E: Mechanism of glutamate receptor desensitization. Nature 2002, 417:245-253.

* The authors demonstrate, on the basis of structural, biochemical and functional data that the agonist-binding domain of GluR2, an AMPA receptor subunit, dimerizes. The dimer interface controls receptor desensitization and provides a binding site for allosteric modulators.

17. Schorge S, Colquhoun D: Studies of NMDA receptor function and stoichiometry with truncated and tandem subunits. J Neurosci 2003, 23:1151-1158.

18. Williams $\mathrm{K}$ : Ifenprodil discriminates subtypes of the $\mathrm{N}$-methyl-D-aspartate receptor: selectivity and mechanisms at recombinant heteromeric receptors. $\mathrm{Mol}$ Pharmacol 1993, 44:851-859.

19. Paoletti $P$, Ascher $P$, Neyton J: High-affinity zinc inhibition of NMDA NR1-NR2A receptors. J Neurosci 1997, 17:5711-5725.

20. Traynelis SF, Burgess MF, Zheng F, Lyuboslavsky P, Powers JL: Control of voltageindependent zinc inhibition of NMDA receptors by the NR1 subunit. J. Neurosci. 1998, 18:6163-6175.

21. Kew JN, Kemp JA: lonotropic and metabotropic glutamate receptor structure and pharmacology. Psychopharmacology (Berl) 2005, 179:4-29.

22. Kinarsky L, Feng B, Skifter DA, Morley RM, Sherman S, Jane DE, Monaghan DT: Identification of subunit- and antagonist-specific amino acid residues in the NMethyl-D-aspartate receptor glutamate-binding pocket. $J$ Pharmacol Exp Ther 2005, 313:1066-1074.

23. Feng B, Morley RM, Jane DE, Monaghan DT: The effect of competitive antagonist chain length on NMDA receptor subunit selectivity. Neuropharmacology 2005, 48:354-359.

24. Liu L, Wong TP, Pozza MF, Lingenhoehl K, Wang Y, Sheng M, Auberson YP, Wang YT: Role of NMDA receptor subtypes in governing the direction of hippocampal synaptic plasticity. Science 2004, 304:1021-1024.

25. Neyton J, Paoletti P: Relating NMDA receptor function to receptor subunit composition: limitations of the pharmacological approach. J Neurosci 2006, 26:1331-1333.

26. Feng B, Tse HW, Skifter DA, Morley R, Jane DE, Monaghan DT: Structure-activity analysis of a novel NR2C/NR2D-preferring NMDA receptor antagonist: 1(phenanthrene-2-carbonyl) piperazine-2,3-dicarboxylic acid. Br J Pharmacol 2004, 141:508-516. 
27. Mayer ML, Ghosal A, Dolman NP, Jane DE: Crystal structures of the kainate receptor GluR5 ligand binding core dimer with novel GluR5-selective antagonists. $J$ Neurosci 2006, 26:2852-2861.

28. Raditsch M, Ruppersberg JP, Kuner T, Gunther W, Schoepfer R, Seeburg PH, Jahn W, Witzemann V: Subunit-specific block of cloned NMDA receptors by argiotoxin636. FEBS Lett 1993, 324:63-66.

29. Chao J, Seiler N, Renault J, Kashiwagi K, Masuko T, Igarashi K, Williams K: N1-dansylspermine and N1-(n-octanesulfonyl)-spermine, novel glutamate receptor antagonists: block and permeation of $\mathrm{N}$-methyl-D-aspartate receptors. $\mathrm{Mol}$ Pharmacol 1997, 51:861-871.

30. Kashiwagi K, Masuko T, Nguyen CD, Kuno T, Tanaka I, Igarashi K, Williams K: Channel blockers acting at N-methyl-D-aspartate receptors: differential effects of mutations in the vestibule and ion channel pore. Mol Pharmacol 2002, 61:533545.

31. Chenard BL, Menniti FS: Antagonists selective for NMDA receptors containing the NR2B subunit. Curr Pharm Des 1999, 5:381-404.

32. Perin-Dureau F, Rachline J, Neyton J, Paoletti P: Mapping the binding site of the neuroprotectant ifenprodil on NMDA receptors. J. Neurosci. 2002, 22:5955-5965.

33. Malherbe P, Mutel V, Broger C, Perin-Dureau F, Kemp JA, Neyton J, Paoletti P, Kew JN: Identification of critical residues in the amino terminal domain of the human NR2B subunit involved in the RO 25-6981 binding pocket. $J$ Pharmacol Exp Ther 2003, 307:897-905.

34. Choi YB, Lipton SA: Identification and mechanism of action of two histidine residues underlying high-affinity $\mathrm{Zn2+}$ inhibition of the NMDA receptor. Neuron 1999, 23:171-180.

35. Rachline J, Perin-Dureau F, Le Goff A, Neyton J, Paoletti P: The micromolar zincbinding domain on the NMDA receptor subunit NR2B. $J$ Neurosci 2005, 25:308317.

36. Frederickson CJ, Suh SW, Silva D, Thompson RB: Importance of zinc in the central nervous system: the zinc-containing neuron. J Nutr 2000, 130:1471S-1483S.

37. Mott DD, Doherty JJ, Zhang S, Washburn MS, Fendley MJ, Lyuboslavsky P, Traynelis SF, Dingledine R: Phenylethanolamines inhibit NMDA receptors by enhancing proton inhibition. Nat Neurosci 1998, 1:659-667.

38. Jin R, Clark S, Weeks AM, Dudman JT, Gouaux E, Partin KM: Mechanism of positive allosteric modulators acting on AMPA receptors. J Neurosci 2005, 25:9027-9036.

39. Balannik V, Menniti FS, Paternain AV, Lerma J, Stern-Bach Y: Molecular mechanism of AMPA receptor noncompetitive antagonism. Neuron 2005, 48:279-288.

40. Hatton CJ, Paoletti P: Modulation of triheteromeric NMDA receptors by $\mathrm{N}$-terminal domain ligands. Neuron 2005, 46:261-274. 
* In this study, the authors combine mutagenesis and pharmacology "tricks" to isolate recombinant NR1/NR2A/NR2B or NR1/NR2A/NR2C triheteromeric NMDARs and characterize their sensitivity to the subunit-selective allosteric modulators $\mathrm{Zn}^{2+}$ and ifenprodil.

41. Kemp JA, McKernan RM: NMDA receptor pathways as drug targets. Nat Neurosci 2002, 5 Suppl:1039-1042.

42. Chizh BA, Headley PM: NMDA antagonists and neuropathic pain--multiple drug targets and multiple uses. Curr Pharm Des 2005, 11:2977-2994.

43. Kew JN, Trube G, Kemp JA: A novel mechanism of activity-dependent NMDA receptor antagonism describes the effect of ifenprodil in rat cultured cortical neurones. J Physiol 1996, 497 ( Pt 3):761-772.

44. Claiborne CF, McCauley JA, Libby BE, Curtis NR, Diggle HJ, Kulagowski JJ, Michelson SR, Anderson KD, Claremon DA, Freidinger RM, et al.: Orally efficacious NR2Bselective NMDA receptor antagonists. Bioorg Med Chem Lett 2003, 13:697-700.

45. Layton ME, Kelly MJ, 3rd, Rodzinak KJ: Recent advances in the development of NR2B subtype-selective NMDA receptor antagonists. Curr Top Med Chem 2006, 6:697-709.

46. Karadottir R, Cavelier $P$, Bergersen LH, Attwell D: NMDA receptors are expressed in oligodendrocytes and activated in ischaemia. Nature 2005, 438:1162-1166.

* Together with 47 and 48, three back-to-back papers which show that NMDARs are expressed in the myelinating processes of oligodendrocytes and are activated under ischemic conditions. Myelin NMDARs have an unusual subunit composition with high level of NR2C and NR3A subunits. These studies strongly suggest that oligodendrocyte NMDARs, in particular those containing NR3A, are promising therapeutic targets for preventing white matter damage.

47. Micu I, Jiang Q, Coderre E, Ridsdale A, Zhang L, Woulfe J, Yin X, Trapp BD, McRory JE, Rehak $R$, et al.: NMDA receptors mediate calcium accumulation in myelin during See $\left[46^{*}\right]$ chemical ischaemia. Nature 2006, 439:988-992.

48. Salter MG, Fern R: NMDA receptors are expressed in developing oligodendrocyte See $\left[46^{*}\right]$ processes and mediate injury. Nature 2005, 438:1167-1171.

49. Lalo U, Pankratov $Y$, Kirchhoff $F$, North RA, Verkhratsky A: NMDA receptors mediate neuron-to-glia signaling in mouse cortical astrocytes. J Neurosci 2006, 26:2673-2683.

* This study demonstrates that cortical astrocytes express functional NMDARs. Similarly to oligodendrocyte NMDARs, astrocyte NMDARs have a low $\mathrm{Mg}^{2+}$ sensitivity indicating that this might be a distinctive property of glial NMDARs.

50. Tsai G, Coyle JT: Glutamatergic mechanisms in schizophrenia. Annu Rev Pharmacol Toxicol 2002, 42:165-179.

51. Mohn AR, Gainetdinov RR, Caron MG, Koller BH: Mice with reduced NMDA receptor expression display behaviors related to schizophrenia. Cell 1999, 98:427-436.

52. Ballard TM, Pauly-Evers M, Higgins GA, Ouagazzal AM, Mutel V, Borroni E, Kemp JA, Bluethmann $\mathrm{H}$, Kew JN: Severe impairment of NMDA receptor function in mice 
carrying targeted point mutations in the glycine binding site results in drugresistant nonhabituating hyperactivity. J Neurosci 2002, 22:6713-6723.

53. Moghaddam B: Bringing order to the glutamate chaos in schizophrenia. Neuron 2003, 40:881-884.

* A good review on the NMDAR hypo-function hypothesis in the pathophysiology of schizophrenia.

54. Hahn CG, Wang HY, Cho DS, Talbot K, Gur RE, Berrettini WH, Bakshi K, Kamins J, Borgmann-Winter KE, Siegel SJ, et al.: Altered neuregulin 1-erbB4 signaling contributes to NMDA receptor hypofunction in schizophrenia. Nat Med 2006, 12:824-828.

55. Lechner SM: Glutamate-based therapeutic approaches: inhibitors of glycine transport. Curr Opin Pharmacol 2006, 6:75-81.

56. Hess SD, Daggett LP, Deal C, Lu CC, Johnson EC, Velicelebi G: Functional characterization of human $\mathrm{N}$-methyl-D-aspartate subtype 1A/2D receptors. $J$ Neurochem 1998, 70:1269-1279.

57. Honer M, Benke D, Laube B, Kuhse J, Heckendorn R, Allgeier H, Angst C, Monyer H, Seeburg PH, Betz $\mathrm{H}$, et al.: Differentiation of glycine antagonist sites of $\mathbf{N}$ methyl-D-aspartate receptor subtypes. Preferential interaction of CGP 61594 with NR1/2B receptors. J Biol Chem 1998, 273:11158-11163.

58. Kuner T, Schoepfer R: Multiple structural elements determine subunit specificity of Mg2+ block in NMDA receptor channels. J Neurosci 1996, 16:3549-3558.

59. Yamakura T, Mori H, Masaki H, Shimoji K, Mishina M: Different sensitivities of NMDA receptor channel subtypes to non-competitive antagonists. Neuroreport 1993, 4:687-690.

60. Bresink I, Benke TA, Collett VJ, Seal AJ, Parsons CG, Henley JM, Collingridge GL: Effects of memantine on recombinant rat NMDA receptors expressed in HEK 293 cells. Br J Pharmacol 1996, 119:195-204.

61. Fischer G, Mutel V, Trube G, Malherbe P, Kew JN, Mohacsi E, Heitz MP, Kemp JA: Ro 25-6981, a highly potent and selective blocker of N-methyl-D-aspartate receptors containing the NR2B subunit. Characterization in vitro. J Pharmacol Exp Ther 1997, 283:1285-1292. 
Figure 1: Potential sites for ligand binding at NMDARs. Most NMDAR are believed to assemble as tetramers associating two NR1 and two NR2 subunits in a "dimer of dimers" quaternary architecture. For clarity, only one of the two NR1/NR2 heterodimers is shown. The extracellular region of each subunit is made of a tandem of bilobate "Venus-flytrap" domains, the N-terminal domain (NTD) followed by the agonist-binding domain (ABD). In the extracellular region, the subunits dimerize at the level of the ABDs and most likely also at the level of the NTDs. The NR2-ABD binds glutamate while NR1-ABD binds the co-agonist glycine (or D-serine). White arrows indicate binding sites for competitive agonists and antagonists. Thick orange arrows indicate sites known to bind allosteric modulators such as endogenous zinc (NR2A and NR2B NTDs) or ifenprodil-like compounds (NR2B-NTD) both acting as non-competitive antagonists. The ion-channel domain also forms binding sites for pore blockers like endogenous $\mathrm{Mg}^{2+}$, MK-801, memantine or ketamine acting as uncompetitive antagonists. Thin orange arrows indicate putative modulatory sites, which could bind either positive or negative allosteric modulators. Note that the only known NMDAR antagonists which display strong subunit selectivity are the NR2-NTD ligands $\mathrm{Zn}^{2+}$, which selectively inhibits NR2A-containing receptors at nanomolar concentrations, and ifenprodil-like compounds which selectively inhibit NR2Bcontaining receptors. 


\section{Acknowledgments}

This work was supported by INSERM and ANR (PP). We thank Boris Barbour and Marc Gielen for comments on the manuscript. 
Table 1

\section{Competitive NMDAR antagonists: subunit selectivity}

\begin{tabular}{|c|c|c|c|c|c|c|c|}
\hline \multicolumn{8}{|c|}{$\mathrm{K}_{\mathrm{i}}(\mu \mathrm{M})$} \\
\hline Agents & binding site & $\mathrm{R} 1 / 2 \mathrm{~A}$ & $\mathrm{R} 1 / 2 \mathrm{~B}$ & $\mathrm{R} 1 / 2 \mathrm{C}$ & $\mathrm{R} 1 / 2 \mathrm{D}$ & selectivity & limitations \\
\hline (R)-AP5 & NR2-ABD & 0.3 & 0.5 & 1.6 & 3.7 & $2 \mathrm{~A} \approx 2 \mathrm{~B}>2 \mathrm{C} \approx 2 \mathrm{D}$ & \\
\hline (R)-AP7 & NR2-ABD & 0.5 & 4.0 & 6.0 & 17 & $2 \mathrm{~A}>2 \mathrm{~B} \approx 2 \mathrm{C}>2 \mathrm{D}$ & \\
\hline PMPA & NR2-ABD & 0.8 & 2.7 & 3.5 & 4.2 & $2 \mathrm{~A}>2 \mathrm{~B} \approx 2 \mathrm{C} \approx 2 \mathrm{D}$ & \\
\hline (R)-CPP & NR2-ABD & 0.04 & 0.3 & 0.6 & 2.0 & $2 \mathrm{~A}>2 \mathrm{~B} \approx 2 \mathrm{C}>2 \mathrm{D}$ & \\
\hline NVP-AAM077 & NR2-ABD & 0.006 & 0.06 & 0.01 & 0.04 & $2 A \approx 2 C>2 B \approx 2 D$ & poor selectivity \\
\hline PPDA & NR2 ABD & 0.6 & 0.3 & 0.1 & 0.1 & $2 \mathrm{D} \approx 2 \mathrm{C}>2 \mathrm{~B} \approx 2 \mathrm{~A}$ & $(\leq 10-$ fold $)$ \\
\hline 7-CKA & NR1-ABD & 0.6 & 0.2 & 0.1 & 0.6 & $2 \mathrm{C} \approx 2 \mathrm{~B}>2 \mathrm{~A} \approx 2 \mathrm{D}$ & \\
\hline 5,7-DCKA & NR1-ABD & 0.03 & 0.05 & 0.2 & 0.09 & $2 \mathrm{~A} \approx 2 \mathrm{~B} \approx 2 \mathrm{D}>2 \mathrm{C}$ & \\
\hline CGP 61594 & NR1-ABD & 0.4 & 0.04 & 0.2 & 0.3 & $2 \mathrm{~B}>2 \mathrm{~A} \approx 2 \mathrm{C} \approx 2 \mathrm{D}$ & \\
\hline
\end{tabular}

$I_{50}$ values determined from the inhibition of NMDAR currents recorded in Xenopus oocytes.

Abbreviations: (R)-AP5, (R)-2-amino-5-phosphonopentanoate; (R)-AP7, (R)-2-amino-7-phosphonoheptanoate; PMPA, (RS)-4-

(phosphonomethyl)-piperazine-2-carboxylic acid; (R)-CPP, (R)-4-(3-phosphonopropyl) piperazine-2-carboxylic acid; NVP-AAM077, [(R)-[(S)-1(4-bromo-phenyl)-ethylamino]-(2,3-dioxo-1,2,3,4-tetrahydroquinoxalin-5-yl)-methyl]-phosphonic acid; PPDA, (2S*,3R*)-1-(phenanthren-2carbonyl)piperazine-2,3-dicarboxylic acid; 7-CKA, 7-chlorokynurenic acid; 5,7-DCKA, 5,7-dichlorokynurenic acid; CGP 61594, ( \pm )-trans-4-[2-(4azidophenyl)acetylamino]-5,7-dichloro-1,2,3,4-tetrahydroquinoline-2-carboxylic acid.

References: for (R)-AP5, (R)-AP7, PMPA and (R)-CPP [23]; for PPDA [26]; for NVP-AAM077 [25,26]; for 7-CI KA [3]; for 5,7-DCKA [56]; for CGP 61594 [57]. 
Table 2

NMDAR channel blockers: subunit selectivity

\begin{tabular}{|c|c|c|c|c|c|c|}
\hline \multirow[b]{2}{*}{ Agents } & \multirow[b]{2}{*}{$\mathrm{R} 1 / 2 \mathrm{~A}$} & \multicolumn{4}{|c|}{$\mathrm{IC}_{50}(\mu \mathrm{M})$ at -60 or $-70 \mathrm{mV}$} & \multirow[b]{2}{*}{ limitations } \\
\hline & & $\mathrm{R} 1 / 2 \mathrm{~B}$ & $\mathrm{R} 1 / 2 \mathrm{C}$ & $\mathrm{R} 1 / 2 \mathrm{D}$ & selectivity & \\
\hline $\mathrm{Mg}^{2+}$ & 20 & 20 & 80 & 80 & $2 \mathrm{~A} \approx 2 \mathrm{~B}>2 \mathrm{C} \approx 2 \mathrm{D})$ & \\
\hline phencyclidine (PCP) & 0.1 & 0.1 & 0.2 & 0.2 & $2 \mathrm{~A} \approx 2 \mathrm{~B} \approx 2 \mathrm{C} \approx 2 \mathrm{D}$ & poor selectivity \\
\hline ketamine & 0.7 & 0.5 & 0.5 & 0.7 & $2 \mathrm{~A} \approx 2 \mathrm{~B} \approx 2 \mathrm{C} \approx 2 \mathrm{D}\}$ & $(\leq 10-$ fold $)$ \\
\hline memantine & 0.9 & 0.8 & nd & 0.5 & $2 A \approx 2 B \approx 2 C \approx 2 D$ & \\
\hline$(+) \mathrm{MK}-801$ & 0.01 & 0.01 & 0.1 & 0.1 & $2 \mathrm{~A} \approx 2 \mathrm{~B}>2 \mathrm{C} \approx 2 \mathrm{D}$ & \\
\hline argiotoxin-636 & 0.009 & 0.005 & 0.46 & nd & $2 A \approx 2 B>>2 C \approx 2 D$ & \\
\hline$N^{1}$-dansyl-spermine & 0.3 & 0.3 & 16 & 13 & 50-fold & \\
\hline
\end{tabular}

$\mathrm{IC}_{50}$ values determined from the inhibition of NMDAR currents recorded in Xenopus oocytes or HEK cells.

References: for $\mathrm{Mg}^{2+}$ [58]; for PCP, ketamine and (+)MK-801 [59]; for memantine [60]; for argiotoxin-636 [28]; for $\mathrm{N}^{1}$-dansyl-spermine [29].

nd: not determined 
Table 3

Subunit-selective NMDAR allosteric inhibitors

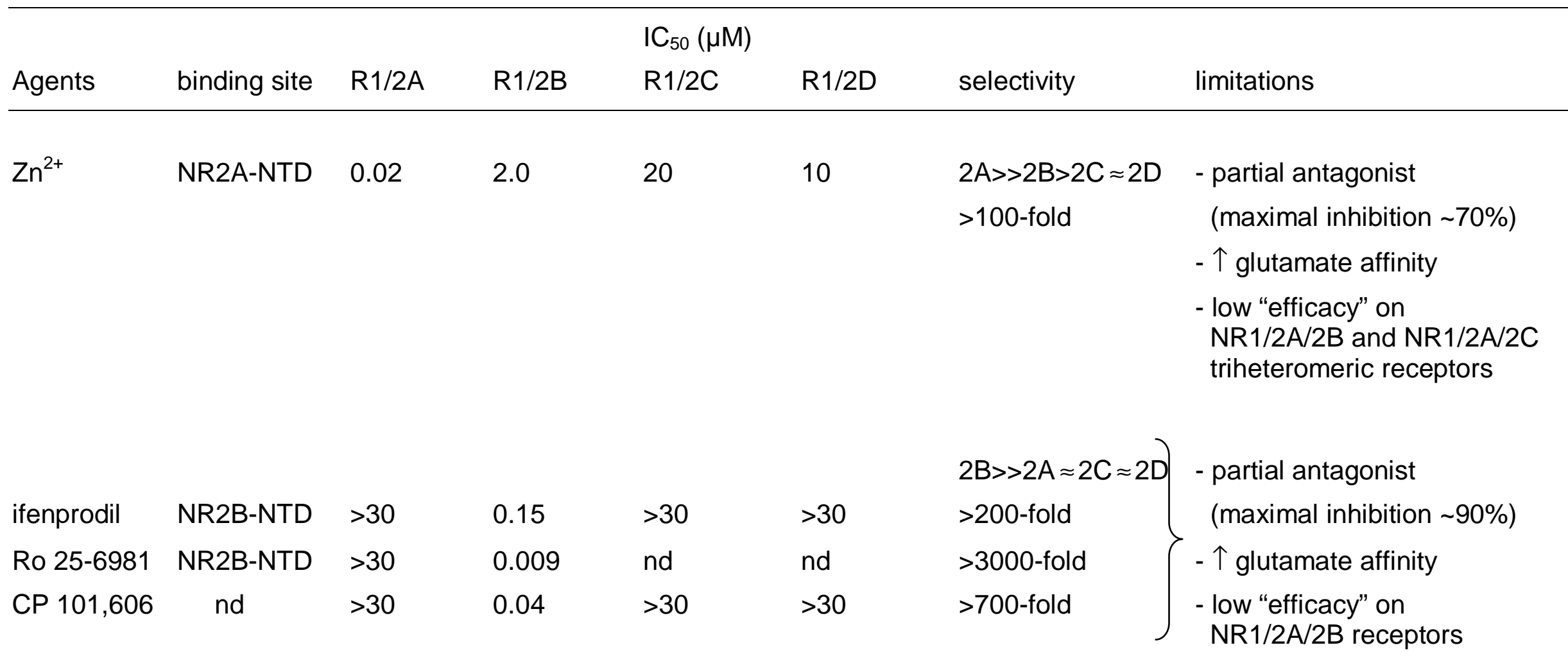

$\mathrm{IC}_{50}$ values determined from the inhibition of NMDAR currents recorded in Xenopus oocytes.

References: for $\mathrm{Zn}^{2+}$ [19,20,35]; for ifenprodil [18,56]; for Ro 25-6981 [61]; for CP 101,606 [37].

nd: not determined 